\section{STIM1, CD36 lingual et perception gustative lipidique}

Souleymane Abdoul-Azize, Gado Dramane, Selvakumar Subramanium, Anne-Marie Simonin, Philippe Besnard, Naim Akhtar Khan
> Selon l'Organisation mondiale de la santé (OMS) [1], l'obésité atteint des proportions épidémiques dans le monde, avec plus de 1,6 milliards d'adultes en surpoids et au moins 500 millions de personnes cliniquement obèses. Globalement, un adulte sur dix est obèse dans le monde. Cette situation a des conséquences importantes en termes de santé publique. En effet, l'obésité, particulièrement l'obésité viscérale, est un facteur de risque de pathologies telles que le diabète de type II, les atteintes cardiovasculaires, I'hypertension et certaines formes de cancers [2].

Bien qu'il existe un déterminisme génétique, les facteurs environnementaux dont l'alimentation jouent un rôle important dans l'apparition de l'obésité [3]. Dans le régime occidental, les lipides alimentaires représentent environ $40 \%$ des apports caloriques journaliers alors que selon les recommandations nutritionnelles, cette proportion devrait être de $35 \%$. Des études comportementales récentes indiquent une préférence spontanée pour les lipides alimentaires chez le rongeur de laboratoire (rat, souris), ainsi que chez I'homme [4]. On a longtemps cru que la perception orosensorielle des lipides alimentaires dépendait uniquement des propriétés texturales et olfactives de ces derniers, mais des découvertes récentes remettent en question ce point de vue restrictif et suggèrent que la gustation joue également un rôle important dans la perception orosensorielle des lipides alimentaires et pourrait donc être impliquée dans la préférence spontanée pour les aliments gras $[4,5]$.

CD36, un récepteur de lipides impliqué dans la détection orosensorielle des lipides alimentaires

La glycoprotéine CD36 est détectée dans différents tissus dont l'épithélium lingual. Elle présente les caractéristiques structurales et fonctionnelles d'un récepteur de lipides gustatifs [5]. En effet, elle lie les acides gras à longue chaîne (AGLC) (nombre de carbones $\geqslant 16$ ) avec une très haute affinité, possède une structure de type récepteur avec une large poche hydrophobe extracellulaire flanquée de deux courts segments transmembranaires orientés dans le cytoplasme. Dans l'épithélium lingual chez la souris [5] et l'homme [6], l'expression du gène codant pour CD36 est strictement restreinte au bourgeon du goût. Quant à la protéine CD36, nous avons récemment montré qu'elle est abondamment exprimée sur la partie apicale de certaines cellules des bourgeons du goût (TRC, taste receptor cells) bordant le pore gustatif de la papille caliciforme chez la souris [5]. Or, il s'avère que cette glycoprotéine transmembranaire est impliquée dans la perception des lipides alimentaires. En effet, l'invalidation du gène codant pour le CD36 diminue de façon drastique la préférence spontanée pour les AGLC chez la souris, sans pour autant affecter la perception du sucré ou de l'amer [5].
Physiologie de la nutrition et toxicologie (NUTox), UMR U866 Inserm, université de Bourgogne/AgroSup Dijon, 6, boulevard Gabriel, 21000 Dijon, France. naim.khan@u-bourgogne.fr

De plus, nous avons démontré chez la souris que, en réponse à l'interaction entre les AGLC et le CD36 au niveau des TRC, le CD36 est couplé à la phosphorylation de tyrosines kinases de la famille Src (Fyn-p ${ }^{59}$ et Yes-p ${ }^{62}$ ) [7]. Nous avons constaté que les AGLC induisent également une augmentation de la concentration de $\mathrm{Ca}^{2+}$ intracelIulaire $\left(\left[\mathrm{Ca}^{2+}\right] \mathrm{i}\right)$ dans les TRC $[8]$. Ce phénomène est strictement dépendant de CD36 car la signalisation calcique est fortement réduite en présence d'un inhibiteur pharmacologique de la liaison des AGLC à CD36 [8]. Cette augmentation du $\left[\mathrm{Ca}^{2+}\right]$ i semble résulter d'une mobilisation du calcium du réticulum endoplasmique (RE) via la production d'inositol triphosphate $\left(\mathrm{IP}_{3}\right)$ et de l'ouverture des canaux SOC (storeoperated calcium) [7]. Cependant, les mécanismes impliqués dans l'ouverture des canaux SOC des cellules gustatives restaient encore non définis.

\section{STIM1, un senseur calcique}

du réticulum endoplasmique

STIMl (stromal interaction molecule l) est une protéine transmembranaire qui joue le rôle de senseur calcique au niveau des citernes du RE $(\rightarrow)$ [14]. La libération calcique à partir du

$(\rightarrow)$ Voir m/s n ${ }^{\circ} 8-9$, août-septembre 2011, page 737

RE entraîne la perte d'ions calcium au niveau des motifs $\varepsilon F$-hands ${ }^{1}$ de STIMl, ce qui provoque sa translocation vers

\footnotetext{
${ }^{1}$ Le domaine EF-hand (helix-loop-helix structural domain)
} est présent dans de nombreuses protéines liant le $\mathrm{Ca}^{2+}$. 


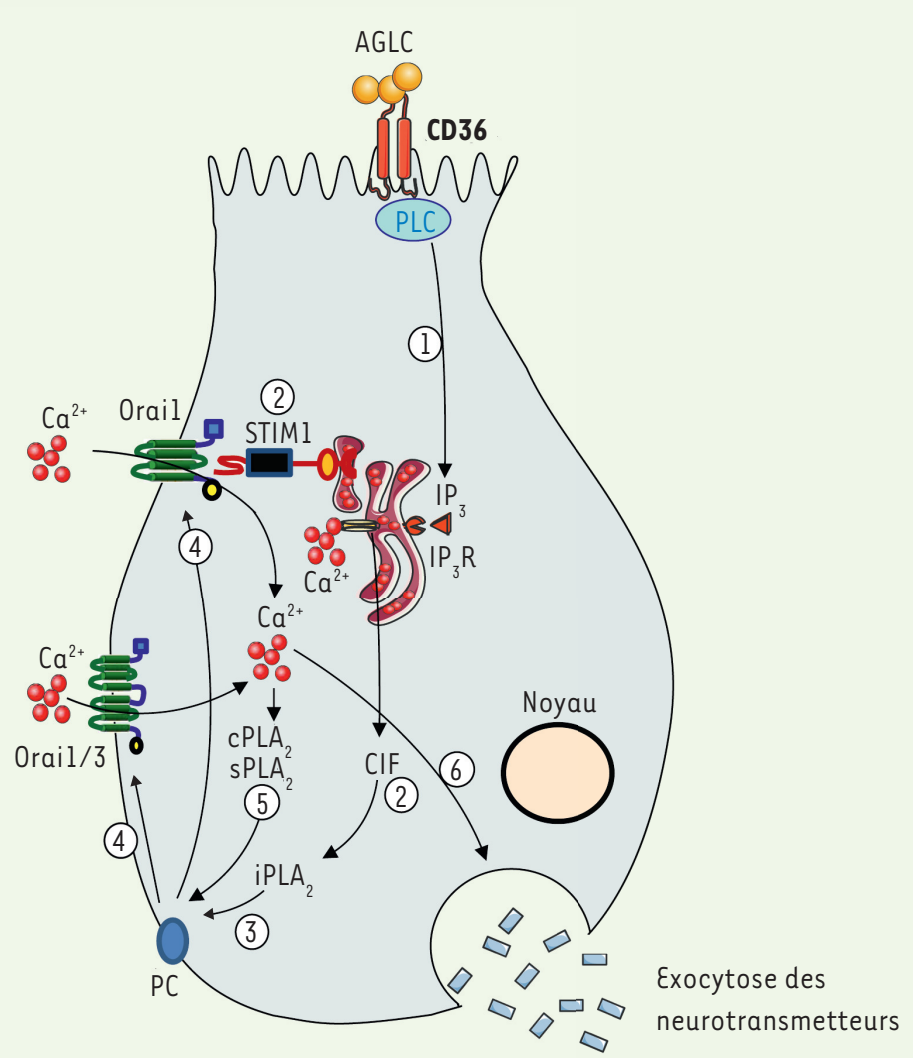

Figure 1. Représentation schématique de l'implication de STIMI dans la signalisation calcique dans les cellules linguales CD36-positives de souris. Un AGLC se fixe sur CD36 puis induit la production d'IP 3 via un mécanisme dépendant de la phopholipase C (PLC) (I). L'IP 3 induit la libération de calcium à partir du RE. Cette déplétion de réserve calcique induit un changement conformationnel de STIMl permettant la libération de CIF dans le cytoplasme (2). Le CIF, à son tour, active $P_{P L A}$ qui donne naissance à la lyso-PC et I'AA (3). Le premier ouvre les canaux calciques ORAll et le second les ORAll/3, permettant l'influx calcique (4). Cet influx calcique va activer $C P L A_{2}$ et $s P L A_{2}$ qui, en agissant sur la phosphatidylcholine (PC) puis en produisant la lyso$P C$ et l'AA, induisent à nouveau l'augmentation de calcium intracellulaire (5). L'élévation de $\mathrm{Ca}^{2+}$ cytoplasmique est responsable de la libération des neurotransmetteurs (6) qui transmettent le message gustatif vers les nerfs afférents. $I P_{3} R$ : récepteur à $l^{\prime} I P_{3}$.

la membrane plasmique où il contrôle l'ouverture de canaux SOC [9].

Si STIMl ouvre les canaux SOC, un facteur contrôlant l'entrée sélective de $\mathrm{Ca}^{2+}$ est nécessaire pour reconstituer la réserve du RE. En 1993, l'équipe de R.Y. Tsein [10], puis en 2008 celle de V.M. Bolotina [9], ont démontré qu'un facteur nommé CIF (calcium influx factor) était impliqué dans la détection du calcium au niveau du RE et dans la reconstitution de son stock calcique. Ainsi, STIMl déclenche la libération du CIF à partir du RE lors de la mobilisation du calcium intracellulaire. Ce rôle physiologique de STIMI est confirmé par l'utilisation des cellules déficientes en STIMI [11].

\section{STIMI orchestre les voies}

de signalisation en aval de CD36 dans les cellules gustatives

Nous avons récemment étudié le rôle de STIMl et du CIF dans l'ouverture des canaux calciques au niveau des papilles gustatives caliciformes chez la souris
[12]. Nous avons montré qu'un AGLC comme l'acide linoléique induit la production d'IP 3 , ce qui permet la mobilisation du calcium à partir du RE et donc la libération de CIF [12]. CIF induit l'activation d'une phospholipase $A_{2}\left(P L A_{2}\right)$ indépendante du calcium ( $\mathrm{iPLA} \mathrm{A}_{2}$ ). II est intéressant de noter que les TRC de souris expriment abondamment $\mathrm{iPLA}_{2}$ VIb, connue pour jouer un rôle clé dans la régulation de l'influx SOC dans différents types cellulaires $[9,11]$. Quand la cellule n'est pas stimulée par la présence exogène d'AGLC, iPLA $A_{2}$ VIb est inactive car associée à la calmoduline (CaM). Nos travaux suggèrent que CIF produit en présence d'AGLC entraîne la dissociation de la CaM et de la iPLA 2 VIb, ce qui l'active. Cette étape permet la libération d'acide arachidonique (AA) et de lysophosphatidylcholine (lyso-PC) à partir de phospholipides (PL) de la membrane plasmique. L'activité du CIF est sous le contrôle strict de STIMl, car CIF purifié à partir des TRC STIM1 ${ }^{-/-}$n'active pas iPLA . Nous avons également démontré que CIF pourrait représenter un facteur universel qui permettrait l'ouverture des canaux calciques en exerçant son action sur la face interne de la membrane plasmique. Ainsi, nous avons injecté CIF, purifié à partir des TRC de souris, dans les lymphocytes $T$ humains et, en utilisant les techniques de patch-clamp, nous avons constaté que ce facteur était capable d'induire une augmentation massive de $\left[\mathrm{Ca}^{2+}\right]$ i via les canaux calciques.

Le lyso-PC provoque l'ouverture des canaux calciques ORAll (calcium release-activated calcium channel protein 1$)$. En effet, la réponse de lyso-PC à l'augmentation du $\left[\mathrm{Ca}^{2+}\right] \mathrm{i}$ est complètement supprimée dans des TRC des souris Orail ${ }^{-/-}$. En ce qui concerne l'AA, il ouvre les canaux calciques hétérodimériques ORAII/3 dans les cellules gustatives lipidiques. En utilisant la technologie de siARN (small interfering $R N A$ ), nous avons démontré que le STIMl agit en amont de l'ouverture des deux types de canaux calciques ORAII et $1 / 3$. En effet, les ARN interférents de 
STIMI abolissent l'ouverture des canaux calciques ORAIl et ORAII/3 dans ces cellules.

Les TRC de souris expriment aussi les $P L A_{2}$ dépendantes du calcium ( $\mathrm{CPLA} 2$ et $s P L A_{2}$ ), et principalement les $s P L A_{2}$ $V$, sPLA 2 lla et cPLA $V_{2}$. L'influx calcique active ces deux familles de $\mathrm{PLA}_{2}$ dépendantes du calcium qui, à leur tour, agissent en synergie sur la phosphatidylcholine membranaire et produisent un influx calcique massif via l'AA et Lyso-PC. CIF est spécifique de $\mathrm{iPLA}_{2}$ et n'a donc aucune action sur $\mathrm{CPLA}_{2}$ et $\mathrm{sPLA}_{2}$.

STIMl est indispensable à la détection orosensorielle des lipides alimentaires Nous avons également montré que l'augmentation massive $\mathrm{du}\left[\mathrm{Ca}^{2+}\right] \mathrm{i}$, via CD36, est impliquée dans l'exocytose de la sérotonine (5-hydroxytryptamine ou 5-HT) par les TCR, ce qui permet le transfert du message lipidique au cerveau via les fibres nerveuses afférentes déclenchant ainsi un comportement stéréotypé (attraction pour les aliments gras).

Il est important de noter que la signalisation calcique dépendante de STIMI est indispensable à la perception orosensorielle des AGLC. En effet, l'invalidation du gène codant pour STIMI s'accompagne d'une chute drastique de la préférence spontanée pour les lipides alimentaires chez des souris soumises à un test de double choix. Ce résultat n'est pas dû à des caractéristiques phénotypiques particulières. $\varepsilon n$ effet, les souris Stiml l-/- $^{-}$sont globalement normales exception faite d'une splénomégalie, cette dernière étant sans influence sur la perception gustative lipidique.

\section{Conclusions}

Nos résultats démontrent pour la première fois que le senseur calcique STIMI du RE joue un rôle de chef d'orchestre dans la signalisation calcique dépendant du CD36 au niveau des cellules gustatives de souris. Une des conséquences physiologiques de cette régulation est l'impact de STIMl dans la perception orosensorielle des lipides alimentaires. Il est possible que des mécanismes similaires soient aussi impliqués dans la perception des autres saveurs.

Notre étude contribue à renforcer l'hypothèse de l'existence d'une sixième modalité gustative dédiée à la détection des lipides alimentaires chez la souris. Des études récentes ont démontré qu'un polymorphisme du gène codant pour CD36, aboutissant à une baisse de l'expression de celui-ci, est responsable d'une augmentation du seuil de détection oral des lipides alimentaires chez l'homme [13]. Ce résultat inédit souligne l'importance du CD36 lingual dans la détection des lipides chez le mammifère. Une meilleure compréhension des mécanismes moléculaires à l'origine de cette régulation pourrait, à terme, déboucher sur de nouvelles stratégies nutritionnelles et/ou pharmacologiques visant à limiter la consommation des aliments riches en graisses et contribuant ainsi à la lutte contre l'obésité. À l'avenir, il serait possible de synthétiser des molécules dites faux lipides qui vont déclencher la même sensation que des lipides alimentaires, mais qui n'auront pas d'apports énergétiques. Ces agents pourront êtres utilisés dans le traitement de l'obésité et des pathologies liées à ce fléau mondial. $\diamond$

STIMl is indispensable for the lingual CD36-mediated $\mathrm{Ca}^{2+}$ signaling and spontaneous preference for fat

\section{REMERCIEMENTS}

Les travaux des auteurs sont financés par le Ministère de l'enseignement supérieur et de la recherche, la région Bourgogne (N.A. Khan), le contrat Agence nationale pour la recherche (ANR-07-PNRA-015), le Centre national interprofessionnel de l'économie laitière (CNIEL) et le Human FATaste program (P. Besnard).

\section{LIENS D'INTÉRÊT}

Les auteurs déclarent n'avoir aucun lien d'intérêt concernant les données publiées dans cet article.

\section{RéFÉRENCES}

1. World health organization. Obesity: preventing and managing the global epidemic. Report of a WHO consultation. World Health Organ Tech Rep Ser 2000 ; 894 : 1-253.

2. Xiao J, Yang W. Weight loss is still an essential intervention in obesity and its complications: a review. J Obes $2012 ; 2012$ : 369097.

3. Bray GA, Paeratakul S, Popkin BM. Dietary fat and obesity: a review of animal, clinical and epidemiological studies. Physiol Behav 2004 ; 83 : 549-55.

4. Mattes RD. Is there a fatty acid taste? Annu Rev Nutr $2009 ; 29: 305-27$.

5. Laugerette F, Passilly-Degrace P, Patris B, et al. CD36 involvement in orosensory detection of dietary lipids, spontaneous fat preference, and digestive secretions. J Clin Invest 2005 ; 115 : 3177-84.

6. Simons PJ, Kummer JA, Luiken JJ, Boon L. Apical CD36 immunolocalization in human and taste buds from circumvallate and foliate papillae. Acta Histochem $2010 ; 113: 839-43$.

7. El-Yassimi A, Hichami A, Besnard P, Khan NA. Linoleic acid induces calcium signaling, Src-kinase phosphorylation and neurotransmitters release in mouse CD36-positive gustatory cells. J Biol Chem 2008 ; 283 : 12949-59.

8. Gaillard D, Laugerette F, Darcel N, et al. The gustatory pathway is involved in CD36-mediated orosensory perception of long-chain fatty acids in the mouse. FASEB J 2008 ; 22 : 1458-68.

9. Bolotina VM. Orai, STIMl and $i P L A_{2}$ : a view from a different perspective. J Physiol 2008 ; 586 : 3035-42.

10. Ramdriamampita C, Tsien RY. Emptying of intracellular $\mathrm{Ca}^{2+}$ stores releases a novel small messenger that stimulates $\mathrm{Ca}^{2+}$ influx. Nature 1993 ; $364: 809-14$.

11. Csutora P, Peter K, Kilic H, et al. Novel role for STIMl as a trigger for calcium influx factor production. J Biol Chem 2008 ; 283 : 14524-31.

12. Dramane G, Abdoul-Azize S, Hichami A, et al. STIMl regulates calcium signaling in taste bud cells and preference for fat in mice. J Clin Invest 2012; 122 : 2267-82.

13. Pepino MY, Love-Gregory L, Klein S, Abumrad NA. The fatty acid translocase gene, CD36, and lingual lipase influence oral sensitivity to fat in obese subjects. J Lipid Res 2012 ; 53 : 561-6.

14. Le Deist F, Capiod T. Immunodéficiences et pathologies associées aux mutations dans STIM/ORAI. Un complexe membranaire au cœur de la signalisation calcique. Med Sci (Paris) 2011 ; 27 : 737-45. 